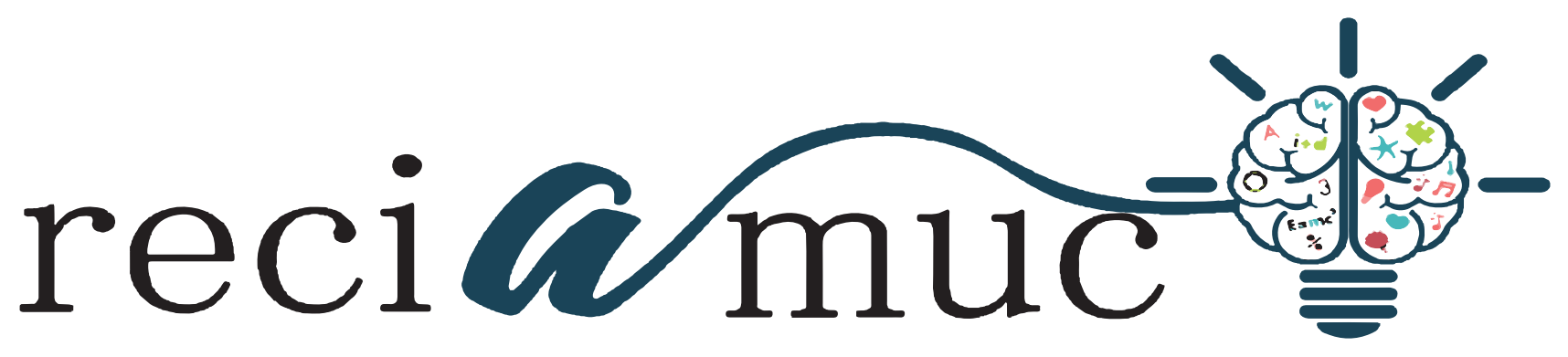

DOI: 10.26820/reciamuc/6.(1).enero.2022.265-274

URL: https://reciamuc.com/index.php/RECIAMUC/article/view/787

EDITORIAL: Saberes del Conocimiento

REVISTA: RECIAMUC

ISSN: 2588-0748

TIPO DE INVESTIGACIÓN: Artículo de revisión

CÓDIGO UNESCO: 5311 Organización y Dirección de Empresas

PAGINAS: 265-274

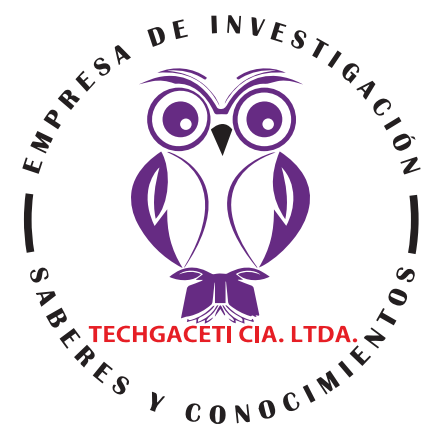

\title{
La influencia de las redes sociales en el proceso de vacunación ciudadana por COVID-19
}

The influence of social networks in the process of citizen vaccination by COVID-19 A influência das redes sociais no processo de vacinação cidadã
pela COVID-19

\section{Carla Estefanía Haro Barre'; Holger Renato Ponce Arreaga2; Freddy Eladio Torres Matamoros ${ }^{3}$}

RECIBIDO: 15/11/2021 ACEPTADO: 05/12/2021 PUBLICADO: 30/01/2022

1. Magister en Ciencias Internacionales y Diplomacia; Licenciada en Ciencias de la Comunicación Social; Docente de la Universidad de Guayaquil; Guayaquil, Ecuador; carla.harob@ug.edu.ec; (D) https://orcid.org/00000002-5087-1299

2. Magister en Administración de Empresas mención Negocios Internacionales; Docente de la Universidad de Guayaquil; Guayaquil, Ecuador; holger.poncea@ug.edu.ec; (D) https://orcid.org/0000-0003-4631-8834

3. Magister en Economía mención en Desarrollo Rural; Economista; Docente de la Universidad de Guayaquil; Guayaquil, Ecuador; freddy.torres-economia@hotmail.com; (DD https://orcid.org/0000-0002-5768-922X

\section{CORRESPONDENCIA}

Carla Estefanía Haro Barre

carla.harob@ug.edu.ec

Guayaquil, Ecuador

() RECIAMUC; Editorial Saberes del Conocimiento, 2022 


\section{RESUMEN}

En población general, un estudio realizado en 19 países encontró una media de reticencia del 28,5\%. En general, la principal preocupación es el riesgo de efectos adversos, basado especialmente en la difusión de noticias falsas. Una revisión de 126 encuestas realizadas durante el año 2020 abarcando 31 países ha mostrado que las actitudes en torno a la vacunación han fluctuado durante el año según la intensidad de la pandemia en el momento de la ejecución de la encuesta, y en general presentan diferencias relacionadas con desigualdades socioeconómicas y afiliaciones políticas, además del deseo de esperar a que otros se vacunen previamente. La metodología utilizada para el presente trabajo de investigación, se enfoca hacia una metodología orientada hacia la necesidad de indagar en forma precisa y coherente una situación. Enmarcada dentro de una revisión bibliográfica de tipo documental, ya que nos vamos a ocupar de temas planteados a nivel teórico como es La influencia de las redes sociales en el proceso de vacunación ciudadana por COVID-19. La técnica para la recolección de datos está constituida por materiales impresos, audiovisuales y electrónicos, estos últimos como Google Académico, PubMed, entre otros. La información aquí obtenida será revisada para su posterior análisis. Hay que aclarar que, dentro de los trabajos de investigación revisados e analizados para desarrollar este texto, no hubo evidencia clara o directa dentro de los instrumentos aplicados, que las personas que tienen una predisposición a vacunarse, hayan tomado esa decisión a causa de las redes sociales o medios de comunicación, ya que es una pregunta que formalmente no se ha realizado. Sin embargo, dentro de las respuestas de las personas que tienen temor a la vacuna por un tema de efecto adverso, seguridad de la vacuna y la rapidez en que fueron creadas, sin duda alguna, son respuestas comunes dentro de los grupos anti vacunas en el mundo, que se han ido difundiendo tanto en los medios de comunicación como las redes sociales.

Palabras clave: Vacunas, Desinformación, Facebook, Twitter, Redes.

\section{ABSTRACT}

Na população geral, um estudo realizado em 19 países encontrou uma relutância média de 28,5\%. Em geral, a principal preocupação é o risco de efeitos adversos, baseado principalmente na disseminação de notícias falsas. Uma revisão de 126 pesquisas realizadas durante o ano de 2020 abrangendo 31 países mostrou que as atitudes em relação à vacinação oscilaram durante o ano dependendo da intensidade da pandemia no momento da execução da pesquisa e, em geral, apresentam diferenças relacionadas às desigualdades socioeconômicas e afiliações políticas, além do desejo de esperar que os outros sejam vacinados antes. A metodologia utilizada para este trabalho de investigação centra-se numa metodologia orientada para a necessidade de investigar uma situação de forma precisa e coerente. Enquadrado numa revisão bibliográfica de tipo documental, uma vez que vamos tratar de questões levantadas a nível teórico, como a influência das redes sociais no processo de vacinação cidadã pela COVID-19. A técnica de coleta de dados é constituída por materiais impressos, audiovisuais e eletrônicos, estes últimos como Google Acadêmico, PubMed, entre outros. As informações aqui obtidas serão analisadas para posterior análise. Deve-se esclarecer que, dentro dos trabalhos de pesquisa revisados e analisados para desenvolver este texto, não houve evidência clara ou direta dentro dos instrumentos aplicados, que pessoas que têm predisposição a serem vacinadas, tomaram essa decisão por causa das redes sociais ou a mídia, já que é uma pergunta que não foi formalmente formulada. No entanto, dentro das respostas das pessoas que têm medo da vacina por causa de um efeito adverso, a segurança das vacinas e a velocidade com que foram criadas, sem dúvida, são respostas comuns dentro dos grupos antivacinas no mundo. mundo, que vêm se espalhando tanto na mídia quanto nas redes sociais.

Keywords: Vaccines, Disinformation, Facebook, Twitter, Networks.

\section{RESUMO}

Na população geral, um estudo realizado em 19 países encontrou uma relutância média de 28,5\%. Em geral, a principal preocupação é o risco de efeitos adversos, baseado principalmente na disseminação de notícias falsas. Uma revisão de 126 pesquisas realizadas durante o ano de 2020 abrangendo 31 países mostrou que as atitudes em relação à vacinação oscilaram durante o ano dependendo da intensidade da pandemia no momento da execução da pesquisa e, em geral, apresentam diferenças relacionadas às desigualdades socioeconômicas e afiliações políticas, além do desejo de esperar que os outros sejam vacinados antes. A metodologia utilizada para este trabalho de investigação centra-se numa metodologia orientada para a necessidade de investigar uma situação de forma precisa e coerente. Enquadrado numa revisão bibliográfica de tipo documental, uma vez que vamos tratar de questões levantadas a nível teórico, como a influência das redes sociais no processo de vacinação cidadã pela COVID-19. A técnica de coleta de dados é constituída por materiais impressos, audiovisuais e eletrônicos, estes últimos como Google Acadêmico, PubMed, entre outros. As informações aqui obtidas serão analisadas para posterior análise. Deve-se esclarecer que, dentro dos trabalhos de pesquisa revisados e analisados para desenvolver este texto, não houve evidência clara ou direta dentro dos instrumentos aplicados, que pessoas que têm predisposição a serem vacinadas, tomaram essa decisão por causa das redes sociais ou a mídia, já que é uma pergunta que não foi formalmente formulada. No entanto, dentro das respostas das pessoas que têm medo da vacina por causa de um efeito adverso, a segurança das vacinas e a velocidade com que foram criadas, sem dúvida, são respostas comuns dentro dos grupos antivacinas no mundo. mundo, que vêm se espalhando tanto na mídia quanto nas redes sociais.

Palavras-chave: Vacinas, Desinformação, Facebook, Twitter, Redes. 


\section{Introducción}

Las vacunas contra el SARS-CoV-2 que se utilizan se han creado con diferentes tecnologías que hasta el momento han demostrado efectividad y han funcionado. La tecnología para vacunar utilizando ácidos nucleicos se viene investigando desde inicios de la década de 1990, por lo que se ha logrado profundizar en el entendimiento de cómo funcionan estas nuevas estrategias para estimular la respuesta inmunitaria. Sin embargo, permanece un factor de riesgo importante en la época de la tecnología digital: la desinformación. Las campañas antivacunación aparecieron desde que inició la vacunación misma, y desde hace décadas han modificado en forma negativa la conducta de las familias para recibir las vacunas. Las aberraciones en los conceptos científicos básicos han llevado a tomar decisiones erróneas a familias enteras. En el año 2019 hubo más de mil casos de sarampión en 31 Estados de los Estados Unidos de América, en familias que principalmente se negaron a recibir la vacuna (Diaz-Badillo, y otros, 2021, pág. 837). La vacuna contra la covid-19 es socialmente muy relevante porque la vuelta a la normalidad sanitaria, económica y social depende de que el mayor número posible de ciudadanos acepte suministrarse este fármaco con el objetivo de obtener cuanto antes la llamada "inmunidad de rebaño", que otorga un eficaz grado de protección en cualquier enfermedad infecciosa y especialmente en una con el alcance de esta pandemia (Polaino, Cárdaba, Cirujano, \& Cambra, 2021, pág. 486).

Desde inicios de la pandemia del 2020, los investigadores mundiales se han esforzado en lograr el desarrollo de la vacunas contra COVID-19, la cual debe ser segura y efectiva para la población a fines de 2020 o principios de 2021 y el éxito de la vacunación COVID-19 depende en gran medida de la aceptación de la vacuna por parte de la población y de esta forma garantizar que se detenga significativamente la propagación de COVID-19, recomendándose un umbral crítico (mínimo) de inmunidad colectiva del $67 \%$ entre la población general; no obstante investigaciones globales recientes sobre la aceptación de la vacuna COVID-19, demuestra que casi el $30 \%$ de los pobladores se niegan a recibir la vacuna una vez que se encuentre disponible, siendo necesario desarrollar estrategias sobre la promoción de la vacuna COVID-19 para lograr su aceptación (Canchanya Cervantes, 2021, pág. 10). En población general, un estudio realizado en 19 países encontró una media de reticencia del 28,5\%. En general, la principal preocupación es el riesgo de efectos adversos, basado especialmente en la difusión de noticias falsas. Una revisión de 126 encuestas realizadas durante el año 2020 abarcando 31 países ha mostrado que las actitudes en torno a la vacunación han fluctuado durante el año según la intensidad de la pandemia en el momento de la ejecución de la encuesta, y en general presentan diferencias relacionadas con desigualdades socioeconómicas y afiliaciones políticas, además del deseo de esperar a que otros se vacunen previamente. Estudios publicados en varios países muestran reticencias entre un $23,1 \%$ en enfermeros/as franceses 11 , hasta un $60 \%$ en enfermeros/as de Hong Kong (Soldevilla, Palma, Hernández, \& Rius, 2021, pág. 21).

Las resistencias de parte de la población hacia determinadas vacunas ya habían llevado a los estudiosos de la comunicación sobre salud y ciencia a fijar su interés en la percepción crítica hacia estas inmunizaciones en el entorno de la Web y muy especialmente, de las redes sociales, debido a su uso creciente para obtener información y opinar sobre temas de salud. Los medios sociales o 2.0 permiten a los usuarios crear, interactuar y compartir contenidos en diferentes formas o formatos de medios, lo que ha facilitado la transmisión de datos y mensajes generalmente breves y con escaso contexto. Por ello, este tipo de comunicación se ha convertido en un ámbito propicio 
para fomentar y expandir determinados posicionamientos que, en muchas ocasiones, sirven para desinformar (Ureta, Fernández, \& i Gras, 2021, pág. 2).

Según la Organización Mundial de Salud (OMS), los programas de vacunación salvan entre 2 y 3 millones de vidas a nivel mundial, su aplicación no solo ha reducido en gran medida los casos de poliomielitis, sarampión, tosferina y otras enfermedades en el mundo, sino que incluso ha erradicado enfermedades como la viruela, evidenciándose así que la finalidad de estos programas no sólo es buscar la inmunidad individual sino la inmunidad colectiva. No obstante, esta situación también ha condicionado que la población le reste importancia al papel preventivo que cumplen las vacunas y perciban una aparente ausencia de riesgo, en especial los jóvenes que desconocen las manifestaciones y la gravedad de las enfermedades que antes eran muy frecuentes. La pandemia de la COVID-19 demanda rapidez en la elaboración y distribución de las vacunas; pero, también demanda la difusión de información con respaldo de la científica. La escasa cultura preventiva puede dificultar el éxito de las campañas de vacunación, por lo tanto, urge tomar acción para comunicarse de manera efectiva y educar a las personas para combatir la desinformación y salvaguardar la salud pública (Huasasquiche, 2021, págs. 1-2).

En las redes sociales, donde prima la libertad de expresión, y a las que muchas personas han acudido en busca de información sobre la COVID-19 ante lo que consideraban una ausencia de información oficial, es donde los grupos que expresan sus opiniones contra las vacunas o que incluso niegan la gravedad o existencia de la COVID-19 han ofrecido contenidos desinformadores. Estos grupos, a menudo, usan argumentos singulares e incluso inventados para atacar a todo aquello relacionado con las vacunas frente a la COVID-19 como, por ejemplo, que la vacuna contendrá "chips" para facilitar el control mental de la pobla- ción. La propaganda extrema de los efectos negativos, inventados o maximizados por estos grupos, usando las redes sociales más extendidas a nivel mundial (Twitter, Youtube, Instagram o Facebook, entre otras, provoca que puedan llegar a un gran número de personas, sembrando la desinformación, llegando a generar miedo e incluso provocando la desconfianza en la información sobre salud proporcionada por las instituciones públicas (Herrera-Peco, Núñez, Jiménez-Gómez, Romero-Magdalena, \& De Gracia, 2021, pág. 2).

Existen diversos estudios relacionados a la aceptación a la vacuna para la COVID-19. Algunos países como China, Ecuador, MaIasia, Indonesia, Corea del Sur, Brasil, Sudáfrica, Dinamarca y Reino Unido, tienen una alta aceptación en rangos que van entre 65 al $97 \%$. Por el contrario, otros países tienen una baja aceptación en rangos entre 55 a $62 \%$, como Rusia y Francia. Esta aceptación varía de acuerdo a factores sociodemográficos, sociológicos, nivel de ingreso, entre otros, los mismos que no los hacen extrapolables a todos los países (Herrera-Añazco, y otros, 2021, pág. 382).

\section{Metodología}

La metodología utilizada para el presente trabajo de investigación, se enfoca hacia una metodología orientada hacia la necesidad de indagar en forma precisa y coherente una situación. Enmarcada dentro de una revisión bibliográfica de tipo documental, ya que nos vamos a ocupar de temas planteados a nivel teórico como es La influencia de las redes sociales en el proceso de vacunación ciudadana por COVID-19. La técnica para la recolección de datos está constituida por materiales impresos, audiovisuales y electrónicos, estos últimos como Google Académico, PubMed, entre otros. La información aquí obtenida será revisada para su posterior análisis. 


\section{Resultados}

Canchanya Cervantes (2021), en su trabajo de investigacion, analiza la percepción social de la aceptación de la vacuna contra la COVID-19 en 1995 usuarios de la red social Facebook, para la recolección de datos fue creado un fan page en las redes sociales, donde fue publicado el cuestionario digital, con fines que los usuarios del Facebook puedan acceder y registrar la información, aceptando previamente el consentimiento informado; para la recopilación de información también fue empleado los grupos WhatsApp. Entre los resultados más relevantes para el objeto de esta investigación se tiene lo siguiente:

- El $88.50 \%$ si aceptaría la vacuna contra la COVID- 19, mientras que el $11.50 \%$ no lo aceptaría.

- En lo que se refiere al conocimiento sobre a vacuna, los usuarios del Facebook indican que la vacuna son anticuerpos $41.50 \%$ y un $32.40 \%$ que son efectivas para evitar el contagio.

- En cuanto al conocimiento sobre el efecto no deseado (de riesgo) de las vacunas contra el COVID- 19 en el organismo, el $35.00 \%$ indicó fiebre, el $27.00 \%$ ningún efecto y el $18.80 \%$ No sabe.

- En cuanto a las razones por la que la gente recibiría la vacuna, se aprecia un $25.90 \%$ dicen que probablemente les daría protección y el $24.40 \%$ por la eficacia de las vacunas COVID-19 respectivamente (págs. 28-31).

Altamirano, y otros (2021), en su trabajo de investigacion, llevaron adelante liderado por Montreal Behavioural Medicine Centre, una encuesta online auto administrable, participando de la misma 3330 adultos argentinos, de entre 18 y 70 años, los resultados mas relevantes fueron los siguientes:

- El 48,9\%, manifestó haber recibido el esquema de vacunación completa.
- Ante la consulta de en qué medida influye en su decisión de vacunación, estos fueron los factores:

- Conveniencia de recibir la vacuna en términos de aplicación, cercanía y gratuidad.

- Recibir la recomendación de mi empleador y/o autoridad laboral para vacunarme.

- Tener información de que la vacuna es segura. - Creer que se posee un alto riesgo de infectarse de $\mathrm{CO}$ VID-19.

- En cuanto a quien influyo en el proceso de su vacunación, un 26\% en gran parte y un $25 \%$ algo su empleador y/o autoridad laboral, un 29\% muy poco, un 19\% para nada.

- El $75 \%$ considera en gran parte que la vacuna es segura, un 17\% algo, un $4 \%$ muy poco.

- En cuanto al riesgo de infección un 49\% lo considera en gran parte, un $26 \%$ algo, un $15 \%$ muy poco, un $9 \%$ para nada y no sabe un $1 \%$.

- En cuanto a la incidencia de las vacunas, un 70,16\% confió en publicaciones científicas que presentaron resultados de eficacia, un $18,03 \%$ al ver resultados de personas que se vacunaron, un 10,49\% al aceptar comunicaciones positivas respecto a la vacuna y la vacunación en medios de comunicación y redes sociales y un 1,31\% por recomendación medica (págs. 6-10).

Ureta, Fernández, \& i Gras (2021), en su trabajo de investigacion, analizaron entre el 14 de diciembre de 2020 y el 7 de febrero de 2021. 62.045 tuits y 258.843 retuits sobre el uso de las etiquetas \#yosimevacuno y \#yomevacuno frente a la de \#yonomevacuno en la red social Twitter. Los resultados mas relevantes fueron los siguientes: 
- El discurso en las redes sociales también ha sido un reflejo de dicha situación. En los inicios del periodo analizado los discursos antivacunas eran dominantes en la conversación en Twitter (Imagen 1), aunque ha ido evolucionando paulatinamente hacia una presencia mayor de los partidarios de la vacunación. Asimismo, han podido identificarse grandes repuntes en la conversación, alrededor de determinados hitos informativos. En el caso del diálogo provacunas, el inicio de la campaña de inmunización el 27 de diciembre de 2020 marcó el momento de mayor actividad en la conversación y alcanzó casi treinta mil retuits en un día. El discurso provacunas también alcanzó un importante repunte coincidiendo con la llegada de millones de vacunas a principios de febrero de 2021. Por el contrario, el discurso antivacunas tuvo uno de sus puntos álgidos también con el inicio de la campaña de vacunación, aunque su impacto total no llegó alcanzar a la mitad del discurso en favor de su uso.

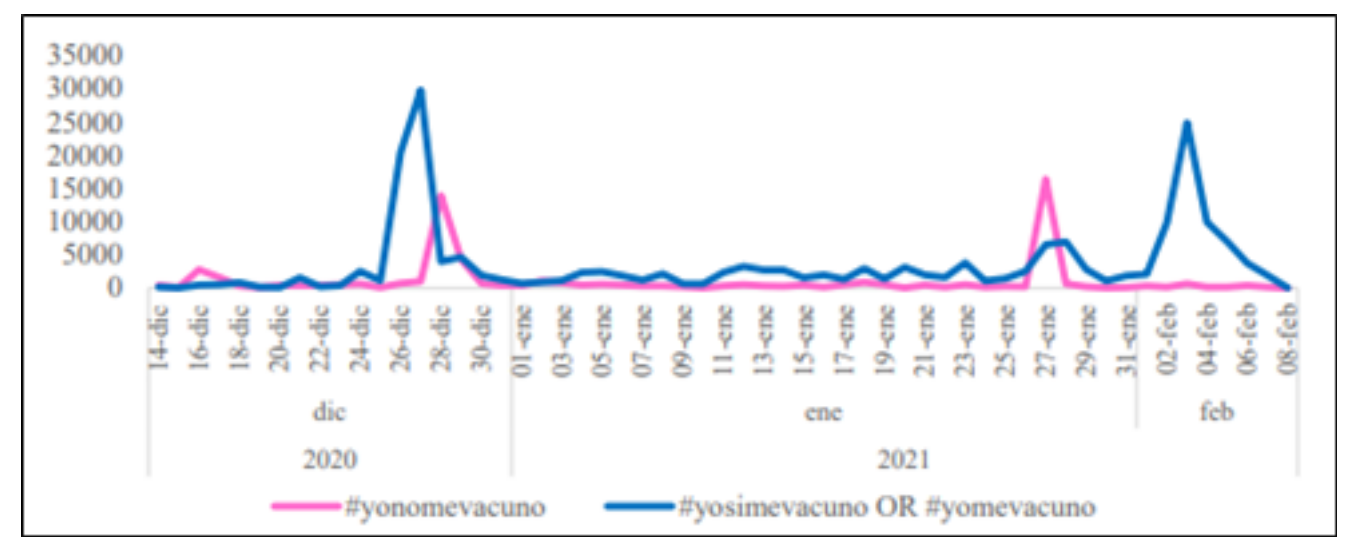

Imagen 1. Retuits diarios según grupo de hashtags.

Fuente: (Ureta, Fernández, \& i Gras, 2021).

En total, los tuits que incluyen etiquetas o palabras clave contrarias a las vacunas han tenido en el periodo analizado un volumen relevante en la conversación global, hasta alcanzar el 38,6\% de los mensajes analizados (Imagen 2). Sin embargo, si analizamos los retuits, los favoritos, las respuestas y las citas, podemos observar que los mensajes provacunas se han viralizado con mayor eficacia, hasta alcanzar cotas cercanas al $80 \%$ del diálogo global. Así, los mensajes provacunas han tenido un promedio de 5,2 retuits, el doble que los mensajes antivacunas. Esta superior redistribución de los mensajes favorables indica, por un lado, la presencia de audiencias activas y generadoras de opinión entre los contrarios a las vacunas, frente a una actitud proporcionalmente menos activa pero más redistributiva y mayoritaria de quienes están a favor.

\begin{tabular}{|l|l|l|}
\cline { 2 - 3 } \multicolumn{1}{c|}{} & $\begin{array}{l}\text { YoSiMeVacuno } \\
\text { /YoMeVacuno }\end{array}$ & YoNoMeVacuno \\
\hline Tuits & 38.069 & 23.976 \\
\hline Retuits & 199.258 & 59.585 \\
\hline Favoritos & 785.139 & 239.203 \\
\hline Respuestas & 73.001 & 18.517 \\
\hline Citas & 22.287 & 5.312 \\
\hline
\end{tabular}

Imagen 2. Volumen del discurso.

Fuente: (Ureta, Fernández, \& i Gras, 2021).

Ríos-González (2021), en su trabajo de investigacion, aplico un cuestionario para identificar la predisposición para recibir la vacuna contra el COVID-19 en Paraguay. Dicho instrumento fue diseñado en Google Forms ( $)$, y difundida a nivel nacional a través de redes sociales (Facebook, Twitter, Instagram) y aplicaciones de mensajería (Whatsapp, Telegram), durante las fechas 
de 20 de enero al 08 de febrero del 2021. Los resultados más relevantes fueron los siguientes:

- Del total de los participantes, $369(47,98$ $\%)$ no se encontraban predispuestos a recibir la vacuna contra el COVID-19, y $101(13,13 \%)$ manifestaron que se encuentran inseguros. Entre los motivos de negarse a aplicarse la vacuna 104 $(41,73)$ mencionaron el temor a los efectos secundarios, 49 (13,27 \%) destacaron que el desarrollo fue muy rápido, 44 $(11,92 \%)$ cree que la vacuna no es segura, y el resto otros (no le gusta las vacunas en general, razones religiosas, no cree que el COVID-19 sea real).
- Del total de los participantes del sexo masculino 201 (58,09 \%) manifestaron que no se encontraban seguros 0 no querían recibir la vacuna, al igual que 98 $(12,74 \%)$ de los que participantes con una edad igual o superior a 35 años, 289 $(76,06 \%)$ de los que se encontraban residiendo el interior del país y 103 (38\%) de los pacientes con alguna enfermedad crónica.

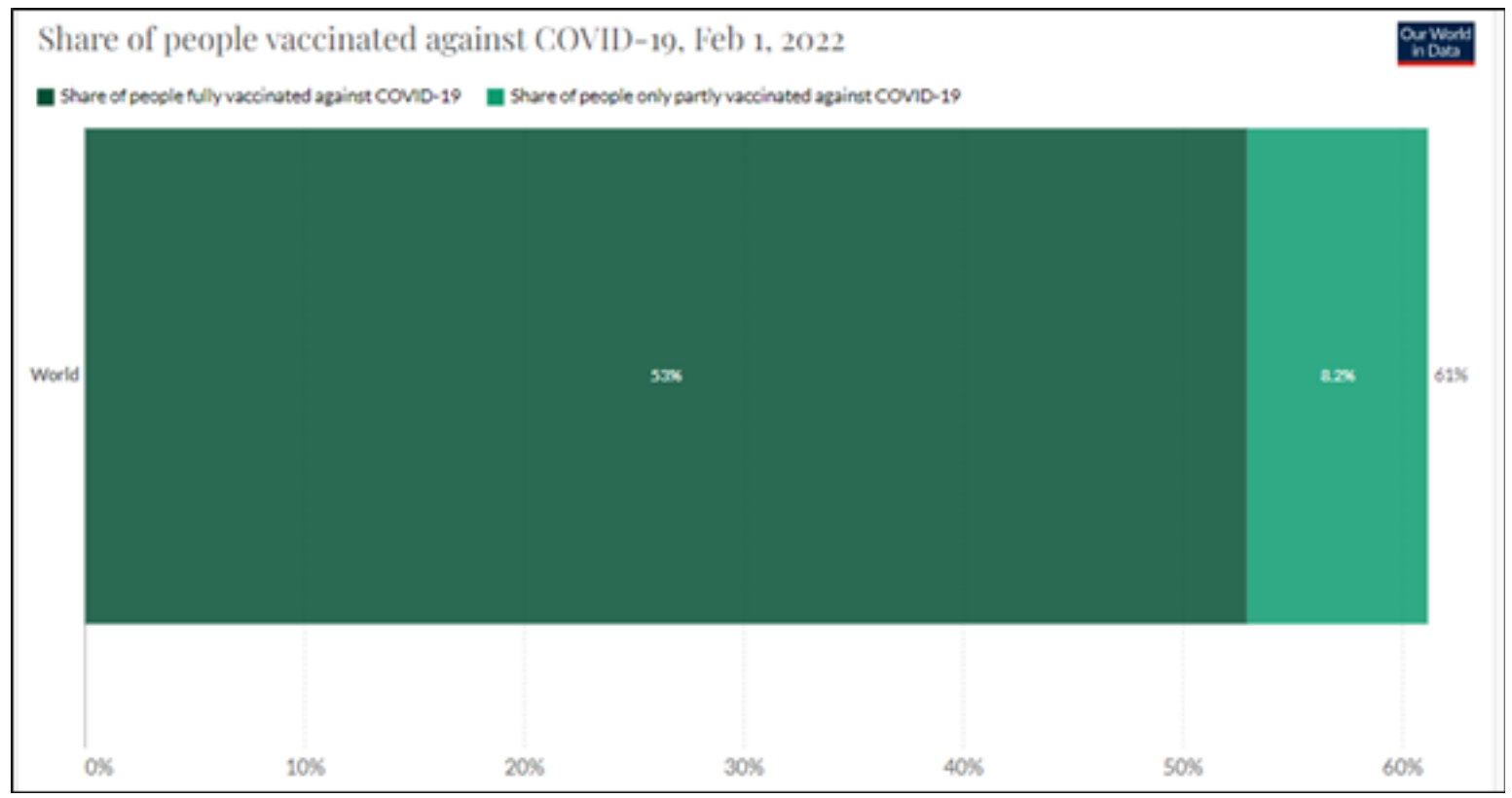

Imagen 3. Personas vacunadas hasta el 01/02/2022 en el mundo.

Fuente: (ourworldindata.org, 2022).

Como se puede observar en la imagen 1. El total de vacunados entre los que tienen el esquema completo de vacunación y los que al menos tienen una dosis alcanza hasta el 01/2/2022 en el mundo un $61,2 \%$. Lo que indicaría que hasta ahora no se ha logrado a nivel mundial la llamada inmunidad de rebaño. 


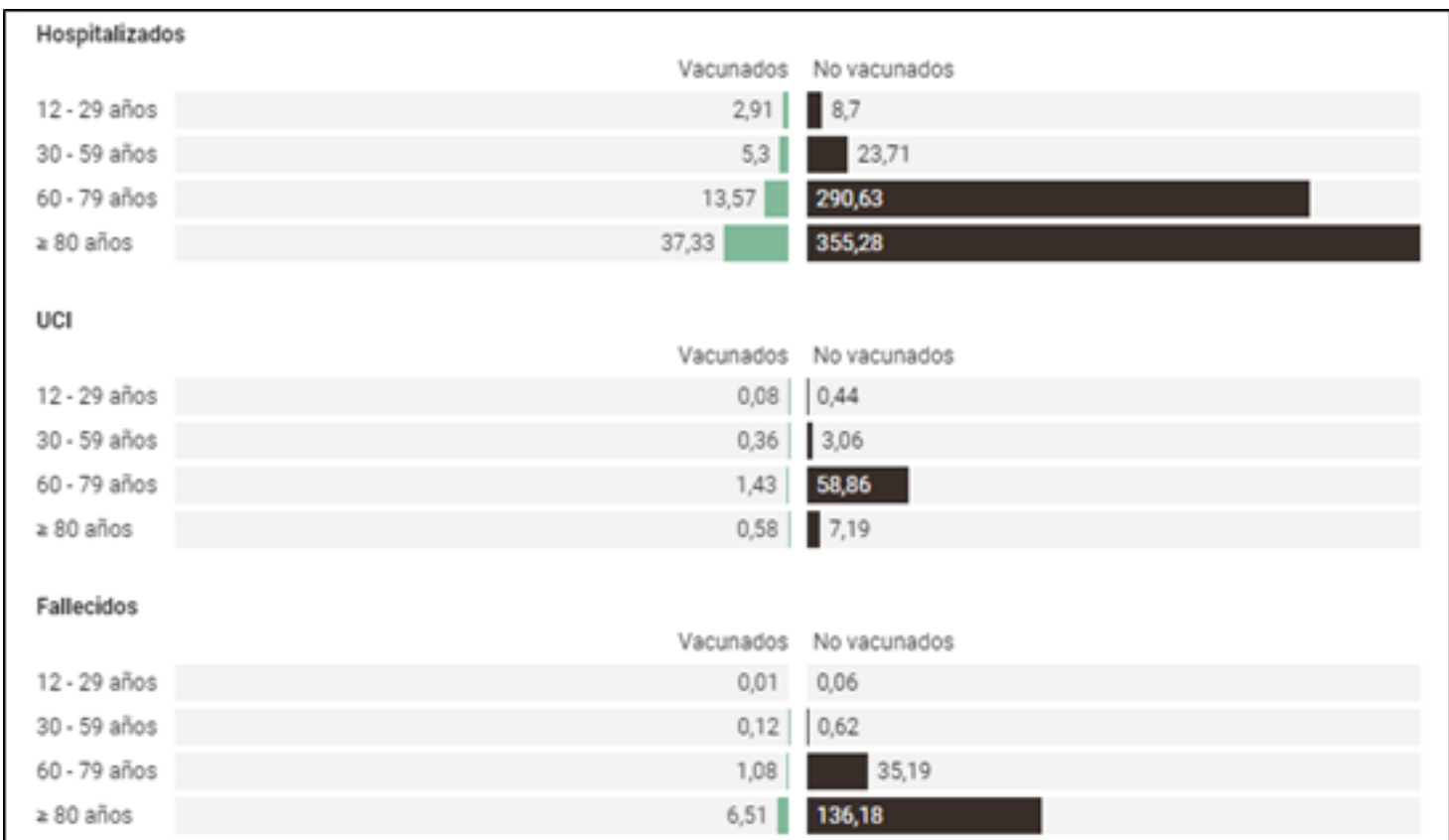

Imagen 4. Hospitalizaciones y defunciones según gravedad y estado de vacunación.

Fuente: (rtve.es, 2022).

En la imagen 4 se observan las diferentes relaciones entre vacunados y no vacunado en España, en todos los casos independientemente de los rangos de edad, en las secciones de hospitalizados, los que ingresaron a $\mathrm{UCl}$ y fallecidos. En donde claramente los porcentajes en todos los casos de estudio superan en muchos casos abrumadoramente a los no vacunados como vacunados como, por ejemplo, los hospitalizados entre 60-79 años el 13,57\% de los vacunados fueron hospitalizados, mientras que el 290,63\% de los no vacunados en el mismo rango de edad fueron hospitalizados.

\begin{tabular}{|c|c|c|c|}
\hline Categoria & Temática & Ejemplo & $\begin{array}{l}\text { Tuits } \\
\text { incluidos } \\
(\mathbf{n} ; \%)\end{array}$ \\
\hline 1 & $\begin{array}{l}\text { Mensajes contrarios } \\
\text { a la vacunación }\end{array}$ & $\begin{array}{c}\text { "YONOMEVACUNO \#yomevacuno. Comienza los LOBBIES } \\
\text { de la Industria a MATAR a los Ancianos y dejar con sindrome } \\
\text { de BELL a los Jóvenes, Sus Armas de llamar BULO a los } \\
\text { que sabemos la Verdad" }\end{array}$ & $3 ; 6 \%$ \\
\hline 2 & $\begin{array}{c}\text { Mensajes } \\
\text { anti-negacionistas }\end{array}$ & $\begin{array}{c}\text { "El ARN de las vacunas NO puede modificar tu ADN. } \\
\text { Las vacunas NO tienen chips de rastreo. Los videos de youtube } \\
\text { y cadenas de whatsapp NO son fuentes confiables. \#YoMeVacuno" }\end{array}$ & $6 ; 12 \%$ \\
\hline 3 & $\begin{array}{l}\text { Información } \\
\text { sobre la gestión } \\
\text { de la vacunación }\end{array}$ & $\begin{array}{l}\text { "La vacunación contra el coronavirus comenzará } \\
\text { en España entre los dias } 27 \text { y } 29 \text { de diciembre. } \\
\text { Se distribuirá en } 50 \text { puntos del pais y se priorizará } \\
\text { su distribución en residencias, centros sociosanitarios } \\
\text { o grandes dependientes. "YoMeVacuno" }\end{array}$ & $8 ; 16 \%$ \\
\hline 4 & $\begin{array}{c}\text { Mensajes } \\
\text { generales sin } \\
\text { contenido especifico }\end{array}$ & "\#YoMeVacuno y dentro de poquito" & $9 ; 18 \%$ \\
\hline 5 & $\begin{array}{l}\text { Vacunación como } \\
\text { responsabilidad } \\
\text { social }\end{array}$ & $\begin{array}{l}\text { "La vacunación es importante por dos aspectos: } \\
\text { 1.Evitar individualmente padecer la enfermedad } \\
\text { 2. Es un gesto de solidaridad para detener la transmisión. } \\
\text { Hoy, la Unión Europea da un paso muy importante para proteger } \\
\text { la salud de la ciudadania. \#YoMeVacuno" }\end{array}$ & $24 ; 48 \%$ \\
\hline
\end{tabular}

Imagen 5. Ejemplos de contenido de tuits individuales (anonimizados).

Fuente: (Herrera-Peco, Núñez, Jiménez-Gómez, Romero-Magdalena, \& De Gracia, 2021). 
En la imagen 5 se observa un comparativo de un plan de difusión llevado a cabo por instituciones del gobierno español con la etiqueta \#YoMeVacuno, en contraste con la etiqueta de los antivacunas \#YONOMEVACUNO en la red social Twitter. Esta claro que los mensajes anti vacunas no han calado dentro de esta muestra, en cambio la bateria de mensajes pro vacunas con mesajes positivos en cuanto retuis ha sido mayor.

\section{Conclusiones}

Hay que aclarar que, dentro de los trabajos de investigación revisados e analizados para desarrollar este texto, no hubo evidencia clara o directa dentro de los instrumentos aplicados, que las personas que tienen una predisposición a vacunarse, hayan tomado esa decisión a causa de las redes sociales o medios de comunicación, ya que es una pregunta que formalmente no se ha realizado. Sin embargo, dentro de las respuestas de las personas que tienen temor a la vacuna por un tema de efecto adverso, seguridad de la vacuna y la rapidez en que fueron creadas, sin duda alguna, son respuestas comunes dentro de los grupos anti vacunas en el mundo, que se han ido difundiendo tanto en los medios de comunicación como las redes sociales.

Esto a causa de la desinformación y las teorías conspirativas que se han tejido alrededor del mundo sobre y como se generó esta pandemia. Pero si está claro que las redes sociales influencian a la población. En el mundo tampoco hay estudios específicos que directamente hayan relacionado la no vacunación por un efecto de una red social. Un 53\% en el mundo tiene I esquema completo de vacunación y un 8,2 al menos se ha colocado una dosis, lo que configura un $61,2 \%$, lo que no quiere decir que un restante $38,8 \%$ sean antivacunas.

\section{Bibliografía}

Altamirano, V. F., Bacon, S., Baró, S., Benítez, D., Caravello, J., Filippa, N., \& Zaracho, R. (2021). Representaciones Sociales sobre las Vacunas y la Vacunación frente al COVID-19. Revista Científica Arbitrada de la Fundación MenteClara, 6(252). doi:https://doi.org/10.32351/rca.v6.252

Canchanya Cervantes, X. (2021). Percepción Social de la Aceptación de la Vacuna Contra la Covid-19 en Usuarios de las Redes Sociales.

Diaz-Badillo, A., Garibay-Nieto, G., Navas-Figueroa, A., Perales-Torres, A., Morales-Gómez, M., \& Lopez-Alvarenga, J. (2021). La vacunación en el contexto de la pandemia de COVID-19. Cirugia y cirujanos, 89(6). doi:http://dx.doi.org/10.24875/ CIRU.21000487

Herrera-Añazco, P., Uyen-Cateriano, A., Urrunaga-Pastor, D., Bendezu-Quispe, G., Toro-Huamanchumo, C., Rodriguez-Morales, A., \& Benites-Zapata, V. (2021). Prevalencia y factores asociados a la intención de vacunación contra la COVID-19 en el Perú. Revista Peruana de Medicina Experimental y Salud Pública(38), 381-390. doi:https:// doi.org/10.17843/rpmesp.2021.383.7446

Herrera-Peco, I., Núñez, C., Jiménez-Gómez, B., Romero-Magdalena, C., \& De Gracia, E. (2021). COVID-19 y vacunación: Análisis del papel de las instituciones públicas en la difusión de información a través de Twitter. Rev Esp Salud Pública, 95(16), 16.

Huasasquiche, Y. B. (2021). La vacunaciòn contra la COVID-19 demanda informaciòn de calidad. Revista Cubana de Enfermerìa, 37(1).

ourworldindata.org. (02 de 02 de 2022). Obtenido de https://ourworldindata.org/covid-vaccinations

Polaino, R. C., Cárdaba, M., Cirujano, E., \& Cambra, U. (2021). Las vacunas contra la Covid en Twitter: redes, mensajes y bots. In Digital media: el papel de las redes sociales en el ecosistema educomunicativo en tiempos de Covid-19. McGraw-Hill Interamericana de España, 487-498.

Ríos-González, C. M. (2021). Predisposición para recibir la vacuna contra el COVID-19 en Paraguay: estudio exploratorio online. Medicina Clínica y Social, 5(2), 119-12. doi:hpps://doi.org/10.52379/ mcs.v5i2.189

rtve.es. (22 de 01 de 2022). Obtenido de https:// www.rtve.es/noticias/20220127/incidencia-covid-segun-vacunacion/2234096.shtml

Soldevilla, P., Palma, D., Hernández, A., \& Rius, C. (2021). Vacuna antiCOVID-19. La otra cara del espejo: la opinión de 5 expertos en vacunas frente a la actitud de los negacionistas y de los antivacunas. Enfermedades Emergentes, 20(11), 20-24. 
Ureta, A. L., Fernández, S., \& i Gras, J. (2021). Desinformación, vacunas y Covid-19: Análisis de la infodemia y la conversación digital en Twitter. Revista Latina de Comunicación Social, 79, 1-18. doi:0.4185/RLCS-2021-1504

\section{CITAR ESTE ARTICULO:}

(c) $\$$ (อ)
BY NC SA

CREATIVE COMMONS RECONOCIMIENTO-NOCOMERCILL-COMPARTIRIGUAL 4.0.

Haro Barre, C. E., Ponce Arreaga, H. R., \& Torres Matamoros, F. E. (2022). La influencia de las redes sociales en el proceso de vacunación ciudadana por COVID-19. RECIAMUC, 6(1), 265-274. https://doi.org/10.26820/reciamuc/6. (1).enero.2022.265-274 